\title{
Ge
}

Santiago Fernández Díez-Picazo*

\section{LOS CONTRATOS QUE OBTIENEN LAS EMPRESAS ESPAÑOLAS EN LAS IFM}

Se presenta un análisis por sectores de los contratos que las empresas españolas han obtenido durante los últimos años en las instituciones financieras multilaterales. Asimismo se intenta averiguar si hay algún sector de grandes adjudicaciones (más de 10 millones de dólares) donde la representatividad de las empresas españolas sea baja.

Palabras clave: adjudicaciones, empresas españolas, ayuda al desarrollo, retornos comerciales, instituciones financieras multilaterales.

Clasificación JEL: F33, L14, O19.

\section{Introducción}

Las instituciones financieras multilaterales (IFM) a las que se refiere este artículo son los grandes bancos de desarrollo: el Banco Mundial (BM), el Banco Interamericano de Desarrollo (BID), el Banco Africano de Desarrollo (BAFD), el Banco Asiático de Desarrollo (BASD), el Banco Centroamericano de Integración Económica (BCIE), el Banco de Desarrollo de América Latina, que mantiene las siglas de su anterior denominación, Corporación Andina de Fomento (CAF), el Banco de Desarrollo del Consejo de Europa (BDCE) y el Banco Europeo de Inversiones (BEI), aunque este último tiene una función más amplia como instrumento financiero de la UE.

Debido al propósito de este artículo, cada una de estas instituciones se tratará como un todo, sin hacer referencia a los componentes internos que pueda tener (por ejemplo, en el caso del BM, la Corporación Financiera Internacional, la Asociación Internacional de Fomento, etcétera).

\footnotetext{
* Consejero Técnico de Fondos Multilaterales.

El autor queda muy reconocido a Juan Francisco Martínez García Subdirector General de Instituciones Financieras Multilaterales, por su estímulo para escribir este artículo, y a todos los compañeros del Ministerio que han hecho observaciones y comentarios.

Versión de mayo de 2015.
}

Estas instituciones conceden préstamos o donaciones a los países prestatarios para la realización de proyectos que contribuyan al avance de sus economías y sus sociedades. Desde la compra de libros para escuelas hasta la construcción de puentes. Desde propuestas de reforma del sistema judicial hasta sistemas de control del tráfico ferroviario. También otorgan financiación y garantías al sector privado, o participan temporalmente en el accionariado de determinadas empresas.

Debe precisarse que este artículo solo analiza una parte de la financiación de las IFM. Por ejemplo, en su año fiscal 2014 (desde el 1 de julio de 2013 al 30 de junio de 2014), el $\mathrm{BM}^{(1)}$ ha financiado 65.600 millones de dólares mediante «préstamos, donaciones, garantías e inversiones en acciones». Sin embargo, los importes de los contratos prior review (con revisión previa por parte del banco antes de firmarlos), que este artículo analiza, solo suman 7.106 millones de dólares para ese año fiscal. Las operaciones de préstamos a Gobiernos fueron 39.500 millones de dólares, de los cuales $\triangleright$

(1) Annual Report 2014. The World Bank. http://www.worldbank.org/ en/about/annual-report 
10.500 millones de dólares fueron préstamos de políticas (aportaciones directas a los presupuestos de esos países). Con la CAF ocurre algo similar.

Los países prestatarios presentan proyectos a la institución. Aquellos para los que se aprueba la financiación son licitados por el país con la supervisión de la IFM. A esta licitación pueden presentarse, en principio, empresas de cualquier país miembro de la institución, aunque en algunos casos se permite que se presenten empresas de cualquier nacionalidad.

\section{Sectores preferentes}

De los respectivos sitios en Internet (ver con detalle en las referencias bibliográficas las direcciones exactas, que no siempre son obvias) se han obtenido datos de los períodos siguientes:

- BID: de 2000 a 2014.

- BM: de 2000 a febrero de 2015.

- BASD: de 2009 a 2014.

- BAFD: del 1 de enero de 2000 al 16 de abril de 2014 (el 09/03/2015 todavía no hay datos detallados del resto de 2014).

Con estos datos, las empresas españolas han obtenido 1.865 contratos por un valor total de 8.269 millones de dólares. Los trabajos son sumamente variados: desde la mejora de carreteras en Uzbekistán hasta una investigación sobre el lavado de manos en Panamá.

En todo este artículo se emplearán dólares norteamericanos como unidad monetaria, pues en ella están los datos originales, y aunque sería posible la transformación a euros año a año, la fuerte variación que han experimentado estas divisas en el período estudiado podría introducir distorsiones. No se analizará el corporate procurement (licitaciones para las propias necesidades de la IFM).

Para el estudio se han dividido en 17 los sectores de adjudicación: Administración Pública, agricultura (que comprende también ganadería, acuicultura, pesca y silvicultura), agua, construcción, educación, energía, estudios, finanzas, industria, informática, medio ambiente, otros, protección contra inundaciones, residuos, salud, transporte y turismo.
El Gráfico 1 muestra que, si se atiende al número de adjudicaciones, está bastante repartido entre estos sectores. El sector grande de la izquierda corresponde a «otros» (generalmente asistencias técnicas de importes reducidos cuya descripción sucinta, como servicios de aprovisionamiento o consejo en la gestión no permite asignarlos con más precisión) y el de la derecha, a energía.

Cuando se habla de «empresas españolas» debe entenderse «empresas con sede en España». Si una empresa española participa en un consorcio con empresas de otros países, el dato de la IFM puede que adjudique todo el importe del contrato a una sola empresa del consorcio, en vez de asignarlo por porcentajes de participación².

Otras veces las adjudicaciones se realizan a filiales en el país de empresas con la matriz en España, que luego consolidan resultados, de modo que los datos oficiales de IFM suelen ser algo menores. Solo a modo de ejemplo, en el BASD en 2011 las adjudicaciones serían 333,5 millones de dólares si contáramos las de Isolux y Cobra obtenidas a través de sus filiales en la India. No es ese el dato que se incluye en el Cuadro 2 (constan solo 44,04 millones de dólares para el BASD en 2011) porque se pretende trabajar con datos homogéneos, pero es importante tenerlo en cuenta.

Asimismo debe precisarse que, aunque un contrato se obtenga con la intervención de una IFM, puede que esa IFM no financie el importe completo del contrato, sino solamente una parte (el resto puede ser aportado por un banco local, directamente por el país adjudicatario, etcétera). Por ejemplo, en el contrato que obtuvo Eptisa Servicios de Ingeniería en 2014 a través del Banco Asiático de Desarrollo por servicios de consultoría para construcción de capacidad y apoyo institucional, el importe completo del contrato fue de 3.384.777,95 dólares, pero el BASD solo financió 2.704.126,97. En este artículo siempre se tiene en cuenta el importe completo.

\footnotetext{
2 Desempeño de las empresas españolas en 2013. Banco Mundial y Banco Interamericano de Desarrollo. ICEX.
} 

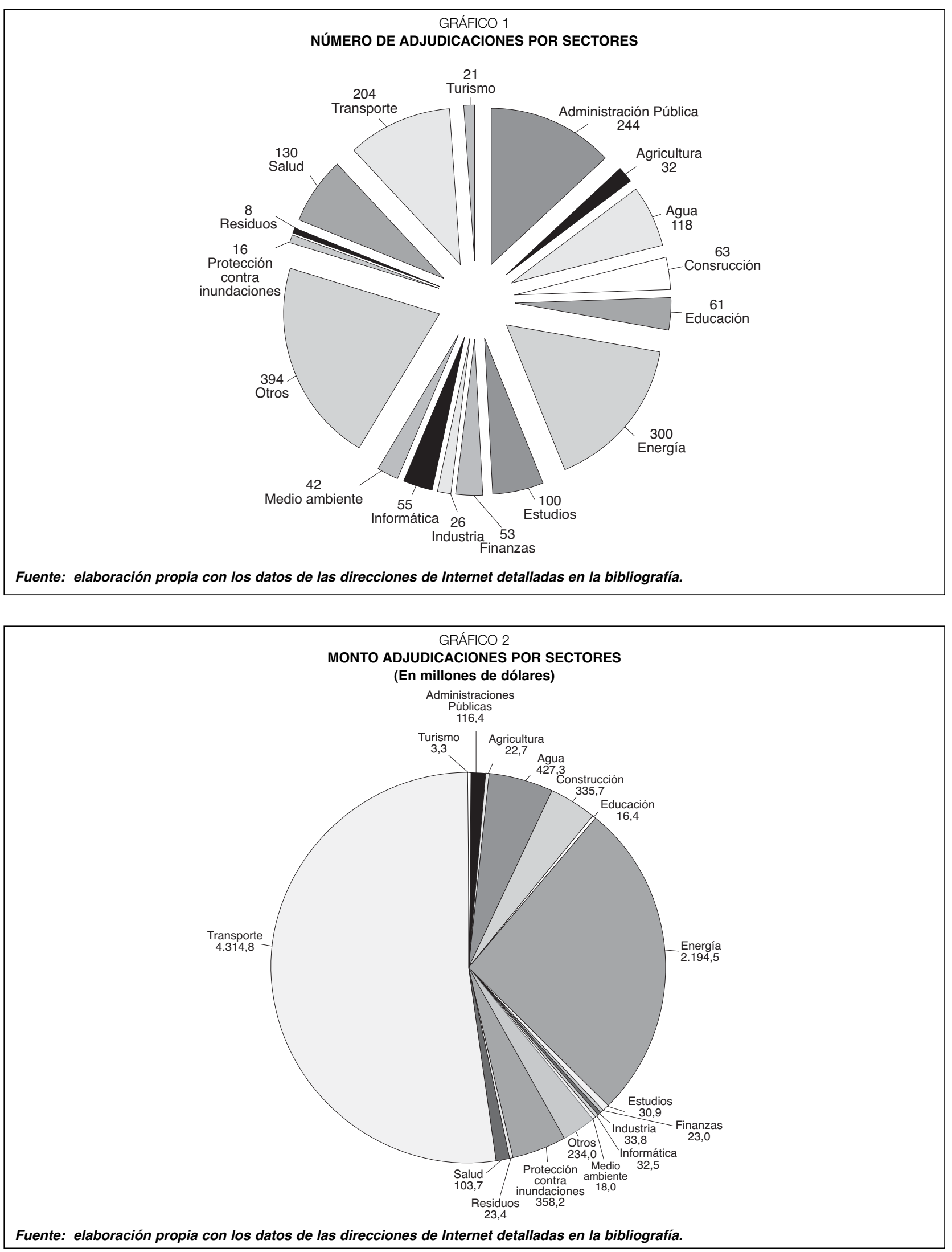

En cambio, si atendemos al monto de los contraen el sector de transporte (más de la mitad, a la tos (Gráfico 2), observamos una fuerte concentración izquierda) y en el de energía (más de un cuarto, $D$ 
a la derecha). Ello se debe a que la construcción de carreteras, puentes, suministro de material rodante (importe medio: más de 21 millones de dólares) líneas eléctricas, centrales eléctricas (importe medio: más de 7 millones de dólares) tiene un importe medio muy superior al de los proyectos para la Administración Pública (477.200 dólares) o del sector «otros» (593.892 dólares).

Si analizamos por bancos los montos de las adjudicaciones (para ello se han tomado solamente las de 2009 a 2013, inclusive, que son los años en que se dispone de datos para los cuatro bancos citados), observamos en el Gráfico 3 una situación interesante.

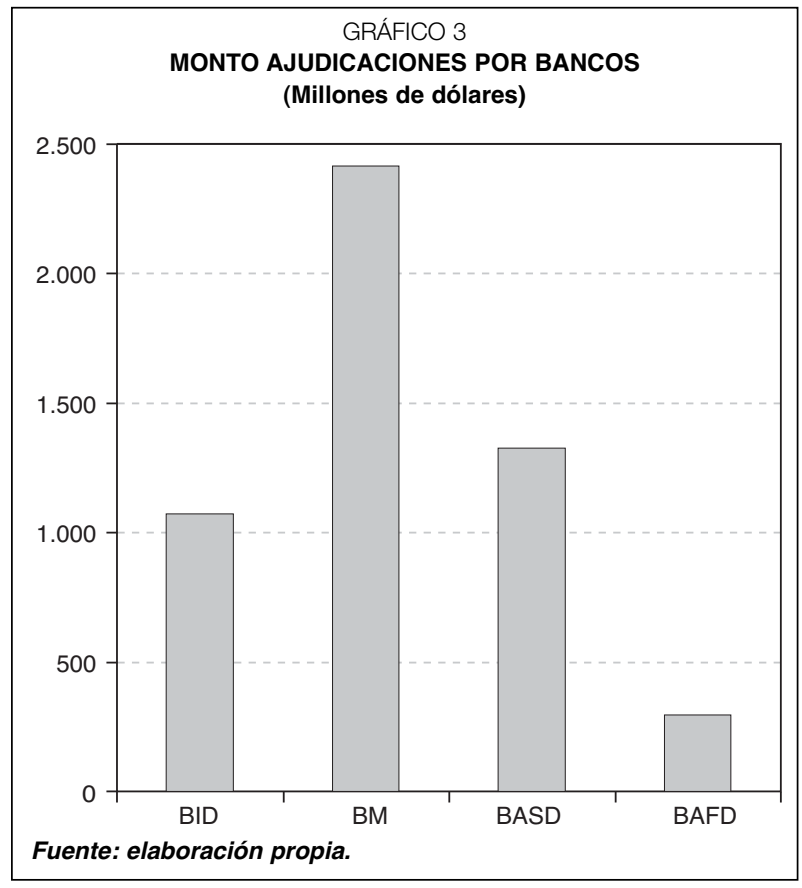

Casi la mitad del importe total se obtiene en el Banco Mundial. Si combinamos esta información con la del Gráfico 4 vemos además que las adjudicaciones más sustanciosas se obtienen en el Banco Asiático de Desarrollo.

En efecto, el Gráfico 5 muestra que la adjudicación media en el Banco Asiático de Desarrollo (25,0 millones de dólares) más que triplica las del Banco Africano de Desarrollo $(8,2)$ y las del Banco Mundial $(8,1)$.

El número de adjudicaciones que obtienen las empresas españolas en cada banco muestra una fuerte dependencia del número total de adjudicaciones.
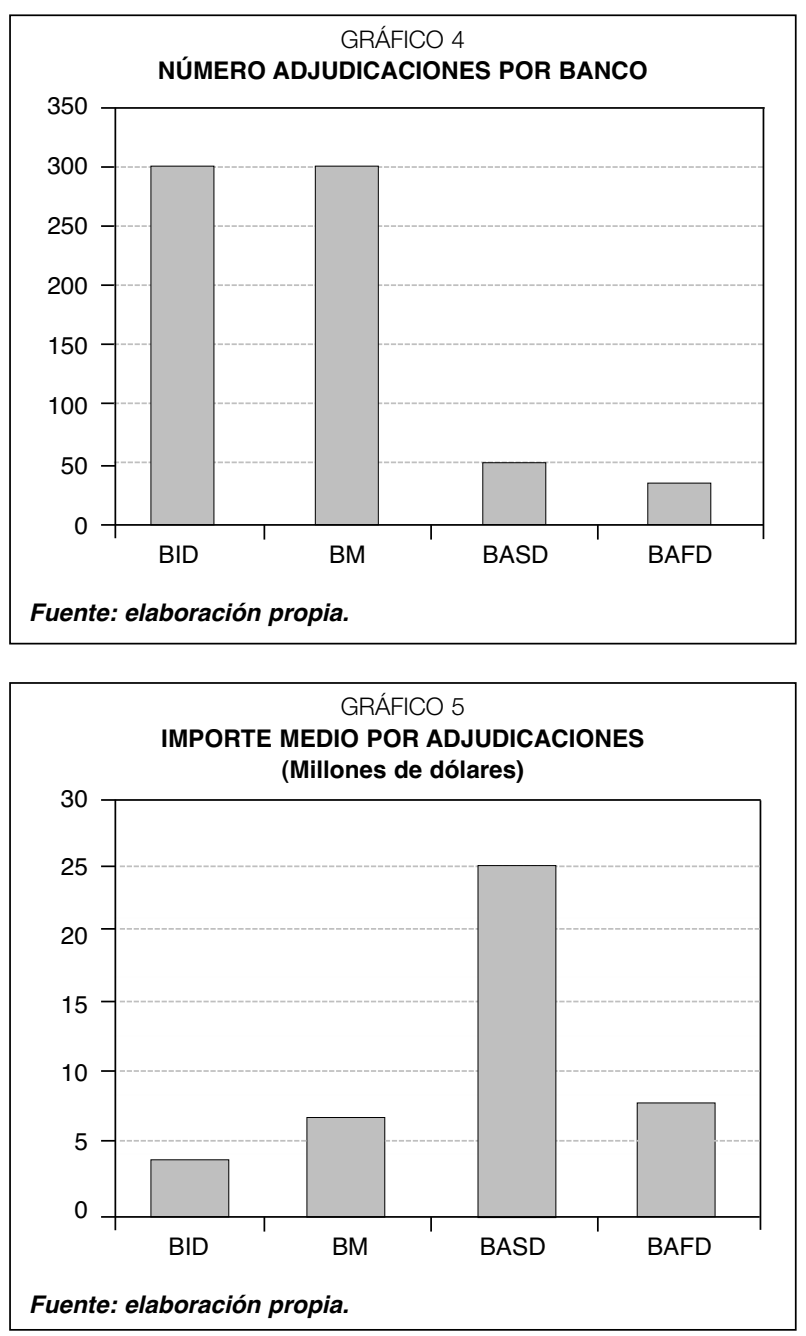

A título de ejemplo, ya que no se dispone de todas las adjudicaciones de estos cuatro bancos desde 2009 a 2013, se han extraído los números totales en 2013: 12.900 en el BID, 11.877 en el BM, 2.035 en el BAFD y 1.349 en el BASD.

Como ya se ha dicho, los datos del BM son contratos con revisión previa, que suponen entre un 15 y un 30 por 100 de sus compromisos anuales. Tampoco incluyen adquisiciones corporativas de la propia institución. Lo mismo se aplica al BID.

El Cuadro 1 muestra los importes, en millones de dólares, de las adjudicaciones anuales a empresas españolas en el período descrito.

El Cuadro 2 muestra el porcentaje del importe que obtienen nuestras empresas respecto al importe anual total de los contratos adjudicados por la IFM (con las salvedades ya descritas). Debajo de las siglas de cada IFM y entre paréntesis está $D$ 


\begin{tabular}{|c|c|c|r|r|}
\hline \multicolumn{7}{|c|}{$\begin{array}{c}\text { CUADRO 1 } \\
\text { ADJUDICACIONES A EMPRESAS ESPAÑOLAS } \\
\text { (Millones de dólares) }\end{array}$} \\
\hline AÑo & BID & BM & BASD & BAFD \\
\hline 2009 & 147,72 & 62,46 & 45,49 & 0,00 \\
\hline 2010 & 29,95 & 53,76 & 38,20 & 9,04 \\
\hline 2011 & 40,27 & 675,20 & 44,04 & 47,98 \\
\hline 2012 & 125,44 & 735,37 & 517,14 & 153,45 \\
\hline 2013 & 748,80 & 799,41 & 398,87 & 111,99 \\
\hline
\end{tabular}

\begin{tabular}{|c|c|c|c|c|}
\hline \multicolumn{5}{|c|}{$\begin{array}{c}\text { CUADRO } 2 \\
\text { PORCENTAJE IMPORTE EMPRESAS ESPAÑOLAS RESPECTO } \\
\text { A ADJUDICACIONES TOTALES DEL BANCO }\end{array}$} \\
\hline $\begin{array}{c}\text { AÑO } \\
\text { (part.) }\end{array}$ & $\begin{array}{c}\text { BID } \\
(1,97)\end{array}$ & $\begin{array}{c}\text { BM } \\
(\mathbf{1}, \mathbf{8 4})\end{array}$ & $\begin{array}{l}\text { BASD } \\
(0,34)\end{array}$ & $\begin{array}{l}\text { BAFD } \\
(1,09)\end{array}$ \\
\hline 2009 & 0,95 & 0,50 & 0,22 & 0,00 \\
\hline 2010 & 0,24 & 0,41 & 0,20 & 0,41 \\
\hline 2011 & 0,37 & 4,64 & 0,20 & 1,93 \\
\hline 2012 & 3,28 & 4,94 & 4,12 & 7,23 \\
\hline 2013 & 11,77 & 7,14 & 4,79 & 4,04 \\
\hline
\end{tabular}

el porcentaje de participación de España en su capital (última cifra disponible en marzo de 2015).

Por ejemplo, España tiene una participación del 0,34 por 100 en el capital del Banco Asiático de desarrollo. Se puede apreciar que, desde unos volúmenes mínimos de adjudicaciones en 2009 , se va ascendiendo hasta otros muy respetables en 2013, que se mantienen claramente por encima de la participación de España en el capital de estas IFM.

En cuanto al análisis por países, las empresas españolas han obtenido 100 adjudicaciones en grupos de países y 1.765 en 117 países individuales. Los tres donde mayor número han obtenido (más de cien en cada caso) son: República Dominicana, Nicaragua y Honduras. Los de superiores montos han sido Brasil (2.324 millones de dólares), India (744 millones de dólares) y Marruecos (582 millones de dólares). En el territorio de Kosovo, no reconocido por España como país, las empresas españolas han obtenido (siempre del BM, porque el BID, BAFD y BASD no conceden créditos a países europeos, salvo el caso del BASD para Georgia, Armenia y Azerbayán) contratos por importe de 128.728 dólares.

\section{Eficacia en la obtención de contratos}

Teniendo en cuenta el anterior análisis por países y que el Banco Mundial tiene 188 Estados miembros, surge de inmediato la pregunta: ¿hay algún país donde las empresas españolas no estén obteniendo adjudicaciones?

Lo primero que debe precisarse es que los miembros de un banco de desarrollo suelen dividirse en países prestatarios, a los que la institución financia (a través de préstamos o donaciones), y países no prestatarios, a los que no financia, porque ya han alcanzado un nivel de desarrollo suficiente. En los casos particulares del BCIE, del BDCE y de la CAF, todos los países pueden ser prestatarios.

Esto quiere decir que si las empresas españolas no obtienen adjudicaciones, por ejemplo del BID en EEUU, no hay que preocuparse, porque allí no se dan. Pero si no las obtuvieran en Haití, sí que debería mirarse por qué (aunque con este país, se puede estar tranquilo: han obtenido 21 adjudicaciones por un importe total superior a los 20 millones de dólares).

Por tanto, hay que extraer todas las adjudicaciones de BM, BAFD, BASD y BID, ver en qué países se han desarrollado los proyectos adjudicados, y hallar si en alguno de ellos, fuera de los 117 países individuales citados, hay adjudicaciones importantes.

Debido a la dificultad de extracción de los datos (en el BASD, para cada año, hay que descargar 60 archivos Excel; en el BID el sistema permite extraer de una vez un máximo de mil adjudicaciones, lo que implica que para sacar todas las de un año han de hacerse más de treinta extracciones; en el BM, cuando el resultado de una búsqueda supere los 15.000 registros, pueden no descargarse todos...) esta parte específica del estudio se ha hecho solo para los años 2013 y 2014 (en el caso del BAFD, del 1 de enero de 2013 al 16 de abril de 2014), tomando siempre como fecha la de firma del contrato (el Banco Mundial sigue el año fiscal norteamericano, de modo que el año fiscal 2014 va desde el 1 de julio de 2013 al 30 de junio de 2014). 
Aun con estas restricciones el número de contratos analizados es considerable: 49.552 .

Ahora se puede responder a la pregunta: entre las adjudicaciones de BM, BAFD, BASD y BID en 2013 y 2014, hay 32 países en los que las empresas españolas llevan al menos desde $2009 \mathrm{sin}$ obtener ninguna adjudicación. Y algunos montos son importantes: por ejemplo, los 166 contratos de los que Papúa Nueva Guinea es país prestatario (es decir, son obras que se ejecutan allí, o servicios que se prestan allí) superan los 489 millones de dólares. Los dos contratos en Namibia, en la práctica uno, la ingeniería, suministro y construcción del terminal de contenedores de la bahía de Wallwis, (que se llevó la empresa China Harbour Engineering) rebasan los 274 millones de dólares.

Los restantes países en esa situación son: Botsuana, Liberia, Burkina Faso, Bután, Sudán del Sur, Burundi, República del Congo, República Centroafricana, Lesoto, Togo, Islas Salomón, Samoa, Birmania, Vanuatu, Comores, Fiyi, Yibuti, Sudán, Palaos, Estados Federados de Micronesia, Somalia, Gabón, Santo Tomé y Príncipe, Nauru, Islas Marshall, Islas Cook, Mauricio, Antigua y Barbuda, San Cristóbal y Nieves, y, finalmente, Dominica (pequeño país de las Antillas Menores, de unos 72.000 habitantes, que no debe confundirse con la República Dominicana).

En todo caso, la suma de los montos 2013-2014 en esos países donde empresas españolas llevan al menos desde 2009 sin obtener adjudicaciones (ya sea porque no se presenten a la licitación, o porque se presenten, pero no la ganen) de bancos de desarrollo (1.995 millones de dólares) es un porcentaje muy reducido (3,42 por 100) de la suma de las adjudicaciones BM-BAFD-BASDBID en todos los países (58.395 millones de dólares).

Además, los importes de los 333 (solo un 0,67 por 100 de los 49.552) contratos adjudicados a empresas españolas suman 3.248 millones de dólares, un 5,56 por 100 de los 58.395 millones de dólares a que ascienden los contratos concedidos a empresas de todos los países. Esto quiere decir que el importe medio logrado por una empresa española (9,75 millones de dólares) es casi nueve veces mayor que la media considerando todos los países (1,18 millones de dólares).

Una posible interpretación es que las empresas españolas no tendrían interés en presentarse a licitaciones de IFM para países con los que existe escaso vínculo comercial. Esto explicaría los casos, por ejemplo, de Palaos o Nauru: según las estadísticas del comercio exterior (http://datacomex.comercio.es), desde el año 2000 no consta una sola exportación a Palaos, y solamente una, en 2013, de 509 dólares, a Nauru. Pero no aclara otros como Liberia, con más de 72 millones de dólares exportados en el período indicado.

Sin embargo, otra visión es que las exportaciones comerciales tradicionales nunca han sido un factor explicativo de las expresiones de interés en proyectos de IFM, precisamente porque, en países con escasa vinculación comercial, presentarse a uno de estos proyectos es una buena forma de establecer una cabeza de puente, aprovechando una licitación en principio justa y objetiva, y el pago garantizado que supone la financiación por una IFM.

Según los comentarios recibidos a una primera versión de este artículo, otro factor que podría explicar esta falta de adjudicaciones a empresas españolas en algunos países sería la corrupción. Por ejemplo, Papúa Nueva Guinea es «uno de los países más corruptos del mundo» [ 145 de 175 en el Índice de Corrupción de Transparencia Internacional, https://www.transparency.org/country\#PNG, el 10 de abril de 2015]. En Liberia «existe comercio bilateral exiguo y volátil. Hubo una primera misión comercial al país en el 2013, no hay oficina comercial española (se lleva desde Accra), las pocas operaciones puntuales son ventas de barcos, corrupción elevada [94 de 175], etcétera. Sin mencionar Sudán del Sur [171 de 175], Bután [30 de 175, cuando en 2014 España está peor, 37. Si las empresas españolas no obtienen adjudicaciones de IFM en Bután, no es por la corrupción], Botsuana [31 de 175, ídem], donde podríamos encontrar razones muy similares». 


\section{4. ¿Se están perdiendo oportunidades?}

Es decir ¿hay algún grupo de grandes contratos (más de 10 millones de dólares) que las empresas españolas no estén obteniendo?

De los casi cincuenta mil contratos considerados, 1.074 son por un importe superior a los $10 \mathrm{mi}-$ llones de dólares. Aunque solo son el 2,17 por 100 de los 49.552 antedichos, la suma de sus importes supone el 66,57 por 100 del importe total de 58.395 millones de dólares.

Prácticamente todos, con alguna excepción irrelevante que detallaré un poco más adelante, caen en los sectores donde las empresas españolas obtienen numerosos contratos: construcción de carreteras, puentes y autopistas; gestión de cuencas para evitar inundaciones; ferrocarriles; suministro de agua y alcantarillado; y tendido de redes eléctricas.

A título de ejemplo, los cinco mayores proyectos adjudicados por BM, BAFD, BASD o BID en 20132014 son:

1. La planta de ciclo combinado de Talimarján, en Uzbekistán (822 millones de dólares), que se adjudicó a Hyundai.

2. La red regional de alta tensión de Tayikistán (558 millones de dólares), que se concedió a Alsthom.

3. Obras eléctricas en Venezuela (475 millones de dólares), que obtuvo la china Dongfang Electric.

4. El plan de gestión de los recursos naturales de Túnez, que fue a la tunecina STEG.

5. Un proyecto regional africano de infraestructura de comunicaciones (438 millones de dólares), que recayó en la empresa keniata Dimension Data.

A este respecto conviene destacar que entre los 12 proyectos de importe más elevado hay tres que se adjudicaron a empresas españolas: uno a Acciona Infraestructuras por 398 millones de dólares para la construcción de un puente, un túnel y una autopista elevada en Brasil; otro de iguales características, salvo el importe (313 millones de dólares); y un tercero, a Dragados, por 289 millones de dólares, para obras de protección contra inundaciones en la cuenca del río Odra (Polonia).
Cuantitativamente, los 41 grandes contratos (3,82 por 100 de los 1.074) adjudicados a empresas españolas suman un monto de 2.939 millones de dólares, el 7,56 por 100 de los 38.873 millones de dólares a los que ascienden los de todos los países. El gran contrato medio español es por tanto claramente superior al general (71,69 millones de dólares frente a 54,37 millones de dólares).

El detalle anteriormente mencionado consiste en la construcción de unos laboratorios médicos en Argentina (204 millones de dólares), el desarrollo de la educación primaria en Bangladés (67 millones de dólares) y (aquí viene lo interesante, porque los dos proyectos anteriores son aislados) cuatro proyectos de energía geotérmica en Indonesia, todos del BM, que suman 259 millones de dólares y que se llevaron la japonesa Sumitomo y la indonesia Rekayasa.

Pese a ser España una potencia en energías renovables, tanto en la producción interior como en los contratos que sus empresas obtienen en el exterior, entre los 1.865 contratos obtenidos por empresas españolas a través de los bancos de desarrollo en los períodos citados en el apartado 2 , sectores preferentes, no hay uno solo de energía geotérmica. Los hay de placas solares, centrales termosolares, parques eólicos, campos solares... Y, sin embargo, anualmente «la energía geotérmica está creciendo del 4 al 5 por 100, con unos 700 proyectos desarrollándose en 76 países» ${ }^{3}$. Se ha producido una innovación con la introducción de plantas de ciclo binario y de mejoras en la perforación y extracción. Esto permite la producción cuando la roca caliente subterránea se encuentra a temperaturas relativamente bajas (solo $\left.57^{\circ} \mathrm{C}\right)^{4}$.

Estos cuatro proyectos no son aislados: entre los $49.552-1.074=48.478$ proyectos de menos de 10 millones de dólares hay otros cinco de energía geotérmica.

Este tipo de energía renovable, además de barata, tiene una característica que la convierte en $\triangleright$

\footnotetext{
32014 Annual U.S. \& Global Geothermal Power Production Report. http://geo-energy.org/events/2014\%20Annual\%20US\%20\&\%20 Global\%20Geothermal\%20Power\%20Production\%20Report\%20Final. $p d f$

${ }^{4}$ Geothermal Energy: http://en.wikipedia.org/wiki/Geothermal_energy
} 
una pieza muy adecuada de un mix energético renovable: su continuidad. No es variable como la eólica o la solar: las zonas calientes de donde se extrae siempre están calientes y proporcionan energía todo el tiempo.

Con la lucha actualmente en marcha contra el cambio climático es de esperar que las inversiones en esta energía sigan aumentando. Por ejemplo, en el este de África, concretamente en Kenia, se está tratando de promoverlas. España no puede quedarse fuera. Las empresas españolas líderes en renovables deben potenciar sus divisiones geotérmicas e intentar aprovechar esta oportunidad.

Según otro comentario recibido «en España aún no están en marcha proyectos de este tipo. Se espera que estén funcionando para 2018 (por lo que no existirían referencias necesarias para licitar). De hecho es una línea de trabajo que está desarrollando Invest in Spain ${ }^{5}$, para la atracción de inversión. La experiencia en este campo la tienen principalmente países como USAo Japón que sí que están resultando adjudicatarios de los mismos».

\section{Conclusiones}

Las empresas españolas muestran en los últimos años gran eficacia en la obtención de contratos de IFM. Ha habido un cambio que parece duradero en la posición del sector privado español en este mercado. $Y$ además se ha producido en unas condiciones para las empresas españolas bastante complicadas (alguien podría decir que en parte a causa de ellas).

Las empresas españolas obtienen contratos en prácticamente todos los países donde el monto de adjudicaciones es elevado. Sin embargo, se identifican algunos donde, por el momento, no los consiguen: Papúa Nueva Guinea, Namibia, Botsuana, Liberia, Burkina Faso, Bután y Sudán del Sur, con

\footnotetext{
5 Página de Invest in Spain sobre energías renovables: http://www.investinspain.org/invest/es/sectores/energias-renovables/ descripcion/index.htm/ y página a la que debería conducir el enlace Geotérmica http://www.energias-renovables.com/ articulos-geotermica-30-Geotérmica
}

montos superiores a los 100 millones de dólares sólo sumando los de 2013 y 2014.

Las empresas españolas obtienen contratos en todos los sectores importantes. Como hallazgo marginal, deberían potenciar sus capacidades de presentarse a licitaciones de energía geotérmica.

\section{Bibliografía}

[1] BANCO AFRICANO DE DESARROLLO (2015). Contratos adjudicados. Disponible en: http://www.afdb.org/en/documents/project-relatedprocurement/procurement-statistics/

[2] BANCO ASIÁTICO DE DESARROLLO (2015). Contratos adjudicados. Disponible en: http://www.adb.org/site/business-opportunities/ operational-procurement/consulting/contractsawarded

http://www.adb.org/site/business-opportunities loperational-procurement/goods-services/con tracts-awarded

[3] BANCO INTERAMERICANO DE DESARROLLO (2015). Contratos adjudicados. Disponible en: http://www.iadb.org/es/adquisiciones-de-proyec tos/contratos-adjudicados, $8181 . \mathrm{html}$

[4] BANCO MUNDIAL (2015). Contratos adjudicados. Disponible en:

https://finances.worldbank.org/Procurement/MajorContract-Awards/kdui-wcs3

[5] BANCO MUNDIAL Y BANCO INTERAMERICANO DE DESARROLLO (2015). Desempeño de las empresas españolas en 2013.

[6] GEOTHERMAL ENERGY (2015). Disponible en: http://en.wikipedia.org/wiki/ Geothermal_energy

[7] GEOTHERMAL ENERGY (2015). 2014 Annual U.S. \& Global Geothermal Power Production Report. Disponible en:

http://geo-energy.org/events/2014\%20Annual \%20US\%20\&\%20Global\%20Geothermal\%20P ower\%20Production\%20Report\%20Final.pdf

[8] INVEST IN SPAIN (2015). Energías renovables. Disponible en:

http://www.investinspain.org/invest/es/sectores/ energias-renovables/descripcion/index.html y página a la que debería conducir el enlace Geotérmica http://www.energias-renovables.com/ articulos-geotermica-30-Geotérmica

[9] THE WORLD BANK (2015). Annual Report 2014. Disponible en:

http://www. worldbank.org/en/about/annual-report 